\title{
Compressed Sensing Phase Retrieval
}

\author{
Albert Fannjiang, Wenjing Liao \\ Department of Mathematics \\ UC Davis, CA 95616-8633.
}

\begin{abstract}
Theory of absolute uniqueness for phase retrieval with random illumination is presented. Suitable random illumination eliminates all sources of ambiguity, trivial and nontrivial. As a result, random-illumination-aided phase retrieval algorithms can accurately recover objects with a below-Nyquist sampling rate close to the minimum and reduce the number of iterations by order of magnitude.
\end{abstract}

\section{INTRODUCTION}

Fourier phase retrieval is the inverse problem of recovering the phase information given the Fourier magnitude data which plays an important role in many areas of science and engineering.

Since our motivation is lensless imaging [8], let us consider discrete phase retrieval in two dimensions: Let $\mathbf{n}=$ $\left(n_{1}, n_{2}\right) \in \mathbb{Z}^{2}$ and $\mathbf{z}=\left(z_{1}, z_{2}\right) \in \mathbb{C}^{2}$. Define the multiindex notation $\mathbf{z}^{\mathbf{n}}=z_{1}^{n_{1}} z_{2}^{n_{2}}$. Let $f(\mathbf{n})$ be a complex-valued function defined on $\mathbb{Z}^{2}$ vanishing outside the finite lattice $\mathcal{N}=\{\mathbf{0} \leq \mathbf{n} \leq \mathbf{N}\}$ for $\mathbf{N}=\left(N_{1}, N_{2}\right) \in \mathbb{N}^{2} . \mathbf{m} \leq \mathbf{n}$ mean $m_{j} \leq n_{j}, \forall j$. The discrete phase retrieval problem is to determine $f(\mathbf{n})$ from the knowledge of the magnitude of the Fourier transform

$$
F(\mathbf{w})=\sum_{\mathbf{n}} f(\mathbf{n}) e^{-\mathrm{i} 2 \pi \mathbf{n} \cdot \mathbf{w}}, \quad \mathbf{w}=\left(w_{1}, w_{2}\right) \in[0,1]^{2} .
$$

It is convenient to write

$$
|F(\mathbf{w})|^{2}=\sum_{\mathbf{n}=-\mathbf{N}}^{\mathbf{N}} \mathcal{C}_{f}(\mathbf{n}) e^{-\mathrm{i} 2 \pi \mathbf{n} \cdot \mathbf{w}}
$$

where

$$
\mathcal{C}_{f}(\mathbf{n})=\sum_{\mathbf{m}+\mathbf{n} \in \mathcal{N}} f(\mathbf{m}+\mathbf{n}) f^{*}(\mathbf{m})
$$

is the autocorrelation function of $f$. By Shannon's sampling theorem $\mathcal{C}_{f}$ can be fully extracted from the Fourier

*Corresponding author: fannjiang@math.ucdavis.edu. The research is partially supported by the NSF grant DMS - 0908535. magnitude sampled on the regular grid

$$
\mathcal{L}=\left\{\left(k_{1}, k_{2}\right): k_{j}=0, \frac{1}{2 N_{j}+1}, \cdots, \frac{2 N_{j}}{2 N_{j}+1}\right\}
$$

Then the phase retrieval problem can be stated as determining the object function $f$ from its autocorrelation function $\mathcal{C}_{f}$.

The first central question is that of uniqueness which traditionally has meant uniqueness up to equivalence class of "trivial associates", including constant global phase,

$$
f(\mathbf{n}) \longrightarrow e^{\mathrm{i} \theta} f(\mathbf{n}), \quad \text { for some } \theta \in[0,2 \pi],
$$

spatial shift

$$
f(\mathbf{n}) \longrightarrow f(\mathbf{n} \oplus \mathbf{m}), \quad \text { for some } \mathbf{m} \in \mathbb{Z}^{2}
$$

and conjugate inversion

$$
f(\mathbf{n}) \longrightarrow f^{*}(\mathbf{N}-\mathbf{n} \oplus \mathbf{m})
$$

where $\mathbf{n} \oplus \mathbf{m}=\mathbf{n}+\mathbf{m}\left(\bmod \left(N_{1}+1, N_{2}+1\right)\right)[1,6,7]$. Conjugate inversion produces a twin image which is a main stumbling block for standard phase retrieval algorithms [11].

In our approach [3], we pursue the notation of absolute uniqueness: if two finite objects $f$ and $g$ give rise to the same Fourier magnitude data, then $f=g$ unequivocally. An essential ingredient of our approach is random (phase or amplitude) illumination which is a form of coded-aperture imaging. This turns out to be pertinent to the main focus of the present paper, i.e. to achieve phase retrieval with sampling rate strictly below the Nyquist rate (1).

The rest of the paper is organized as follows. In Section II, we discuss phase retrieval from the perspective of compressed sensing. In Section III, we present the theory of absolute uniqueness. In Section IV, we present our numerical results. We draw conclusions in Section V.

\section{COMPRESSED MEASUREMENTS}

When the autocorrelation function $C_{f}$ is sparse and has, say, $K$ non-zero elements, then, with a high probability, 

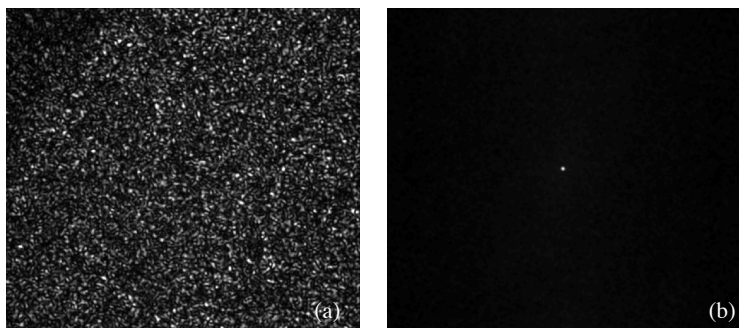

Fig. 2. (a) Encoding speckle pattern. (b) Autocorrelation of the encoding pattern.

Figure 1: (a) Nearly i.i.d. speckle pattern and (b) its autocorrelation produced by a light diffuser (adapted from [5]).

the whole function $|F(\mathbf{w})|^{2}, \mathbf{w} \in \mathcal{L}$ can be recovered by compressed sensing techniques [10] from a sampling subset consisting of $\mathcal{O}\left(K \sum_{j=1}^{2} \log N_{j}\right)$ independent, uniformly distributed points in $\mathcal{L}$ or $[0,1]^{2}$.

If the sequence $f$ has $S \leq|\mathcal{N}|$ nonzero components (i.e. the sparsity equals $S$ ), then it is easy to see that $C_{f}$ has at most $S(S-1) / 2+1$ nonzero components. Hence the whole function $|F(\mathbf{w})|^{2}, \mathbf{w} \in \mathcal{L}$ can be recovered from $\mathcal{O}\left(S^{2} \sum_{j=1}^{2} \log N_{j}\right)$ samples with high probability.

Unfortunately, taking advantage of this standard compressed sensing result would limit the objects of interest to the class of one-dimensional objects. A more powerful approach is to use random illumination to effectively turn the deterministic object into a random object.

Random illumination amounts to replacing the original object $f(\mathbf{n})$ by

$$
\tilde{f}(\mathbf{n})=f(\mathbf{n}) \lambda(\mathbf{n})
$$

where $\lambda(\mathbf{n})$, representing the incident field, is a known sequence of samples of random variables, typically assumed to be independent and identically distributed (i.i.d.), Figure 1.

The most important property about $\lambda(\mathbf{n})$ is that they are continuous random variables with respect to the Lebesgue measure on $\mathbb{S}^{1}$ (the unit circle), $\mathbb{R}$ or $\mathbb{C}$. The case of $\mathbb{S}^{1}$ can be facilitated by a random phase modulator with

$$
\lambda(\mathbf{n})=e^{\mathrm{i} \phi(\mathbf{n})}
$$

where $\phi(\mathbf{n})$ are continuous random variables on $[0,2 \pi]$ while the case of $\mathbb{R}$ can be facilitated by a random amplitude modulator. The Gaussian and binary masks used in [2] are examples of random amplitude illumination. The case of $\mathbb{C}$ involves simultaneously both phase and amplitude modulations.
For a metric of compression, let us define the sampling ratio

$$
\rho=\# \text { Fourier magnitude data/\# image pixels }
$$

following [9]. The Nyquist rate (1) corresponds to $\rho=4$ in two dimensions. The goal of compressed sensing is Fourier phasing with the sampling ratio $\rho<4$.

\section{ABSOLUTE UNIQUENESS}

First we have the following uniqueness result [3].

Theorem 1. Suppose that $f(\mathbf{n})$ is real and nonnegative for every $\mathbf{n}$ and that $\{\lambda(\mathbf{n})\}$ are independent continuous random variables on $\mathbb{S}^{1}$ or $\mathbb{R}$ or $\mathbb{C}$. Then, with probability one, $f$ is determined absolutely uniquely by the Fourier magnitude measurement on the lattice $\mathcal{L}$.

Another widely used constraint is the positivity of both real and imaginary parts of complex-valued objects [9]. For such objects, we have the following result [3].

Theorem 2. Suppose that $f(\mathbf{n})$ has nonnegative real and imaginary parts for every $\mathbf{n}$. Let $S$ be the sparsity (the number of nonzero elements) of the object.

(i) Consider the random phase illumination (4). Suppose that the phases $\phi(\mathbf{n})$ are i.i.d. uniform random variables on $[0,2 \pi]$. Then with probability no less than $1-|\mathcal{N}| 4^{-[S / 2]}$ the object $f$ is absolutely uniquely determined by the Fourier magnitude measurement. Here $[S / 2]$ is the greatest integer less than or equal to $S / 2$.

(ii) Consider the random amplitude illumination with i.i.d. random variables $\{\lambda(\mathbf{n})\} \subset \mathbb{R}$ that are equally likely negative or positive, i.e. $\mathbb{P}\{\lambda(\mathbf{n})>0\}=\mathbb{P}\{\lambda(\mathbf{n})<$ $0\}=1 / 2, \forall \mathbf{n}$. Then with probability no less than $1-$ $2^{-[(S-1) / 2]}|\mathcal{N}|$ the object $f$ is absolutely uniquely determined by the Fourier magnitude measurement on $\mathcal{L}$.

Such results do not hold for the 1-dimensional phasing problem.

Both Theorem 1 and 2 require the Nyquist sampling with $\rho=4$ which appears to be an overestimate. Actual reconstruction with random illumination requires a much smaller $\rho$ as shown below.

\section{NUMERICAL EXPERIMENTS}

The most widely used phase retrieval algorithm is Fienup's [4] Hybrid Input-Output Algorithm (HIO) based on alternating projections in the object domain and the Fourier domain. Given the $k$-th iterate $f_{k}(\mathbf{n})$ the $(k+1)$ st iteration of HIO is given as follows. 


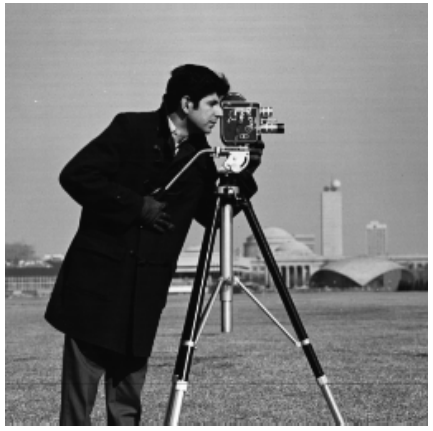

(a)

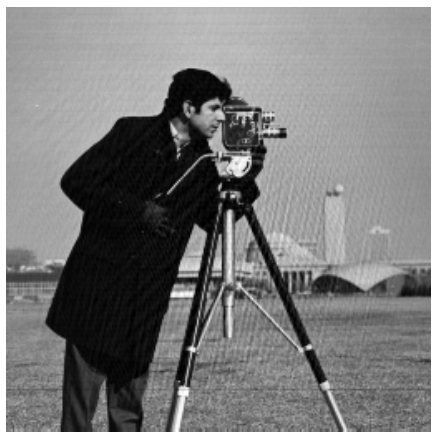

(c)

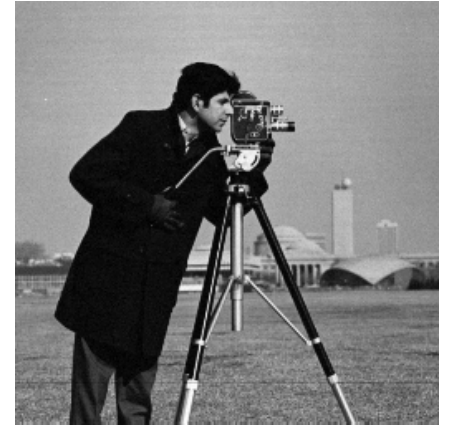

(b)

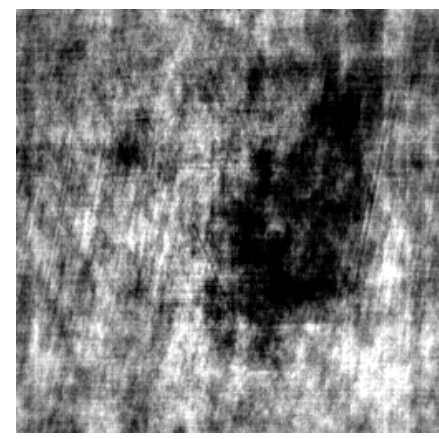

(d)
Figure 2: (a) The original object and reconstructions with (b) random phase illumination, $\rho=1.1$, relative error $\approx$ $2.61 \%$; (c) constant illumination, $\rho=4$, relative error $\approx$ $4.36 \%$; (d) constant illumination, $\rho=2$.

\section{HIO algorithm}

- Update Fourier phase: $F_{k}=\boldsymbol{\Phi} f_{k}=\left|F_{k}(\mathbf{w})\right| e^{i \theta_{k}(\mathbf{w})}$.

- Update Fourier magnitude: $\tilde{F}_{k}(\mathbf{w})=|F(\mathbf{w})| e^{i \theta_{k}(\mathbf{w})}$.

- Inverse Fourier transform $\tilde{f}_{k}=\boldsymbol{\Phi}^{*} \tilde{F}_{k}$.

$f_{k+1}(\mathbf{n})= \begin{cases}\tilde{f}_{k}(\mathbf{n}) & \text { if } \Gamma \text { is satisfied } \\ f_{k}(\mathbf{n})-\beta \tilde{f}_{k}(\mathbf{n}) & \text { if } \Gamma \text { is violated }\end{cases}$ where $\Gamma$ is the object domain constraints.

Here $\boldsymbol{\Phi}$ is the discrete Fourier transform and $\beta$ is the hybridization parameter typically chosen between 0.5 and 1 . In our experiments $\beta=0.9$. The object domain constraints $\Gamma$ may include the support constraint and the object value constraint (e.g. positivity).

One way to introduce the support constraint is by zeropadding with $\rho>1[9]$. Let $\mathcal{N}$ be the original object domain with $\left(N_{1}+1\right)\left(N_{2}+1\right)$ pixels. With $\rho>1$ we enlarge the object domain by adding $(\rho-1)\left(N_{1}+1\right)\left(N_{2}+1\right)$ pixels of value zero around $\mathcal{N}$. Zero-padding can reduce the chance of confusing the object with a shifted object (2). However, zero-padding can not prevent the twin image (3) from getting in the way of reconstruction unless random illumination

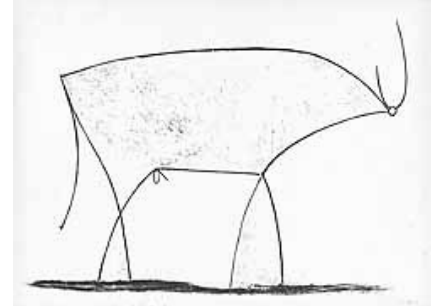

(a)

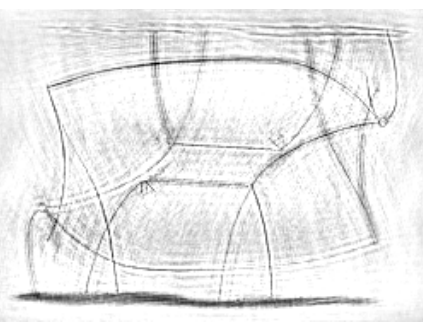

(c)

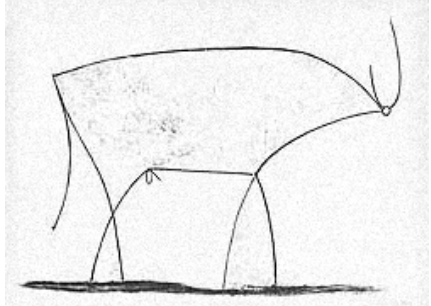

(b)

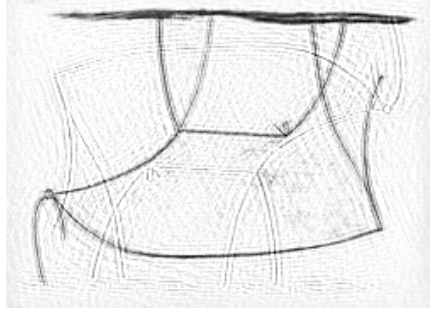

(d)
Figure 3: (a) The original object and reconstructions with (b) random phase illumination, $\rho=1.1$, relative error $\approx$ $2.97 \%$; (c) constant illumination, $\rho=4$, relative error $\approx$ $13 \%$; (d) constant illumination, $\rho=6,\left\|\hat{f}-f_{\text {twin }}\right\| /\|f\| \approx$ $17 \%$.

is applied.

In the following numerical examples (Figures 2 and 3 ) we show how much reduction in data can be achieved by random phase illumination (4). We apply the algorithm HIO followed by 10 steps of error-reductions [4].

For both examples, with single random phase illumination, mere $100 \mathrm{HIO}$ iterations and the sampling ratio $\rho=1.1$ are sufficient to achieve accurate recovery (less than $3 \%$ relative error) (Figure 2 (b), Figure $3(\mathrm{~b})$ ). In contrast, with constant illumination $(\lambda(\mathbf{n})=1, \forall \mathbf{n})$, even 3000 HIO iterations and the sampling ratio $\rho=4$ fails to recover the object due to the interference of the twin image (3) in some case (Figure 3 (c)) and when the sampling ratio increases to $\rho=6$ the twin image finally overwhelms the true image in the reconstruction (Figure $3(d)$ ). Even in the case where recovery with constant illumination seems successful (after 3000 iterations with $\rho=4$ ), the recovered image is contaminated with an artificial pattern of stripes (Figure $2(\mathrm{c})$ ). If the sampling ratio reduces to $\rho=2$, the quality of recovery deteriorates drastically, again, due to the interference of the twin image (Figure $2(d)$ ).

\section{CONCLUSIONS}

We have developed theory of absolute uniqueness for phase retrieval with random illumination. In addition to 
providing psychological assurance, the absence of (trivial or nontrivial) ambiguity probably contributes in a significant way to the superior qualities of random-illuminationaided phase retrieval algorithms which use far fewer Fourier magnitude data and order of magnitude fewer number of iterations.

By a simple dimension counting, it is easy to see that $\rho=1$ is a lower bound for the minimum sampling rate for phasing real-valued objects. In our examples, we have demonstrated that $\rho=1.1$ is sufficient to accurately recover the real-valued objects with single random phase illumination.

\section{REFERENCES}

[1] Yu. M. Bruck and L. G. Sodin, "On the ambiguity of the image reconstruction problem," Opt. Commun.vol. 30, pp. 304-308, 1979.

[2] E. J. Candès, Y. Eldar, T. Strohmer and V. Voroninski, "Phase retrieval via matrix completion," preprint, August 2011.

[3] A. Fannjiang, "Absolute uniqueness of phase retrieval with random illumination," arXiv:1110.5097v3.

[4] J.R. Fienup, "Phase retrieval algorithms: a comparison," Appl. Opt. vol. 21, pp. 2758-2769, 1982.

[5] J. Garcia, Z. Zalevsky, and D. Fixler, "Synthetic aperture superresolution by speckle pattern projection," Opt. Exp. vol. 13, pp. 6073-6078, 2005.

[6] M. Hayes, "The reconstruction of a multidimensional sequence from the phase or magnitude of its Fourier transform," IEEE Trans. Acoust. Speech Sign. Proc. vol. 30, pp. 140- 154, 1982.

[7] M. H. Hayes and J. H. McClellan. "Reducible Polynomials in More Than One Variable." Proc. IEEE vol. 70(2), pp. 197 198, 1982.

[8] J. Miao, T. Ishikawa, Q. Shen, and T. Earnest, "Extending X-Ray crystallography to allow the imaging of non- crystalline materials, cells and single protein complexes," Annu. Rev. Phys. Chem. vol. 59, pp. 387410, 2008.

[9] J. Miao and D. Sayre, "On possible extensions of X-ray crystallography through diffraction-pattern oversampling," Acta Cryst. $A$ vol. 56, pp. 596-605, 2000.

[10] H. Rauhut, "Stability results for random sampling of sparse trigonometric polynomials," IEEE Trans. Inform. Th. vol. 54, pp. 5661-5670, 2008.

[11] H. Stark, Image Recovery: Theory and Applications. New York: Academic Press, 1987. 